Reprod. Nutr. Dévelop., 1980, 20 (3 B), 779-790.

\title{
Remarques sur les relations entre adultes et stades jeunes chez les insectes sociaux
}

par G. LE MASNE

\author{
Institut de Neurophysiologie ef Psychophysiologie \\ C.N.R.S., I.N.P.-7 \\ 31, chemin Joseph-Aiguier, 13274 Marseille Cedex 2
}

Summary. Comments on the relations between adults and immature stages in social insects.

Parental relations in birds and mammals have been compared to relations between adults and immature stages in the most evolved social insects such as termites, social wasps, ants and social bees. These relations in insect societies present an original pattern which is probably more complex than the parental relations in vertebrates.

Il est utile de comparer $\left({ }^{1}\right)$ les relations parentales, et plus généralement les rapports entre jeunes et adultes, tels qu'on les observe d'une part chez les Vertébrés supérieurs (Oiseaux ef Mammifères), d'auire part chez les Insectes et en particulier les Insectes sociaux. Les remarques présentées ici tendront surtout à faire apparaître les différences, c'est-à-dire à montrer combien les Sociétés d'Insectes offrent, pour les relations entre individus adultes et stades jeunes, par comparaison avec les faits connus chez les Vertébrés, un « modèle » original et qui est peut-être, au total, beaucoup plus complexe.

D'autres exposés, dans le même Colloque (Vancassel et Forasté, 1980 ; Krafft et Horel, 1980), apportent une documentation d'une part sur les relations mère-jeune chez les Forficules - chez qui les rapports entre individus correspondent peut-être à l'aurore de la vie sociale - d'autre part chez les Araignées, qui offrent, dans plusieurs lignées, les stades successifs d'une évolution de l'organisation sociale. Je me limiterai ici, à côté de l'exposé de Jaisson (1980) sur les Hyménoptères sociaux, à quelques indications concernant les Sociétés d'Insectes les plus évoluées, qu'on parle de sociétés supérieures (Le Masne, 1952) ou d'espèces eusociales (Wilson, 1971) : celles des Termites, des Guêpes sociales, des Fourmis et des Abeilles sociales.

En ce qui concerne les soins donnés aux jeunes dans les sociétés de Termites, nous disposons (cf. Grassé, 1949 ; Noirot, 1952) d'informations :

- sur le léchage des œufs, qui est indispensable à leur survie (comme c'esł déjà le cas chez les Forficules : of. Vancassel, 1980) ;

(1) Au sens vrai du mot : c'est-à-dire de rechercher, sans aucun a priori, les points communs et les différences entre deux espèces ou ensembles d'espèces. 
- sur le transport des plus jeunes larves, qui se déplacent peu par elles-mêmes à l'intérieur du nid (dans les cas, peu fréquents, de migration d'une partie de la société, les jeunes larves et aussi une partie des larves âgées sont portées dans les mandibules des ouvriers adultes : Grassé et Noirot, 1951),

- sur l'aide apportée par les adultes lors des mues : cette aide paraît nécessaire chez les Termites inférieurs (Calotermes: Noirot, 1952 ; Reticulitermes : Buchli, 1950).

Nous avons surtouł, grâce à l'emploi des radio-isotopes mélangés aux aliments, des informations sur les échanges de nourriture entre individus jeunes et adultes, soit de bouche à bouche (voie stomodéale), soil d'anus à bouche (échange proctodéal) : cf. Grassé ef Noirot, 1945 ; Grassé, 1949 ; Noirot, 1952 ; Gösswald ef Kloft, 1958 ; Alibert, 1959, 1960, 1963, 1969 ; McMahan, 1963, 1969... Ces échanges, on le sait, ont une grande importance quantitative, et sont tout à fait nécessaires à la survie des individus et de la société - non seulement parce que, comme chez les autres Insectes sociaux, seule une partie des membres de la société sortent du nid pour la récolte d'aliments, mais aussi pour d'autres raisons qu'il serait trop long de rappeler ici : par exemple chez les Termites xylophages, la nécessité des échanges proctodéaux pour l'acquisition de l'indispensable faune de Flagellés symbiotiques, ou pour la recontamination des individus après les mues (Grassé et Noirot, 1945).

Les travaux de Alibert en particulier (loc. cit.) ont apporté une série de données de grand intérêł sur les modifications quantitatives des échanges alimentaires entre couple royal et stades jeunes, à mesure que progresse la fondation de la société, et dans la société plus âgée.

Mais si nous avons ainsi des données quantitatives solides sur les échanges alimentaires entre Termites adultes et jeunes, par contre l'étude des postures, des mouvements, des stimulations qui précèdent ef accompagnent les échanges n'a jamais été poussée très loin chez les Termites. Une analyse précise manque.

L'étude comparative, chez des espèces appartenant à des familles de Termites inégalement évoluées, montre que la dépendance des stades jeunes par rapport aux individus âgés est d'autant plus développée et se prolonge d'autant plus qu'on étudie des espèces plus évoluées (Noirot, 1952). Il faut toutefois rappeler ici que les larves de Termites, Insectes « à métamorphoses incomplètes », sont, dès leur naissance, pourvues de pattes ; certes, les larves du premier stade se déplacent peu par elles-mêmes (cf. plus haut), mais les larves plus âgées ef les nymphes pourvues de " fourreaux alaires » se déplacent activement dans le nid ; et les stades jeunes, chez les Termites, ont fondamentalement la même structure que les individus plus âgés, et en partie les mêmes organes.

Au contraire, dans les autres sociétés d'Insectes (celles des Guêpes, des Fourmis et des Abeilles - tous Insectes à métamorphoses complètes), les larves sonf dépourvues de pattes et dépendent entièrement des adultes ; les nymphes, bien sûr, sont immobiles, et souvent enfermées dans un cocon. La structure anatomique des larves diffère de manière profonde de celle des adultes.

Chez les Guêpes sociales, comme chez les Abeilles sociales, les larves sont logées une par une dans des alvéoles étroits, qui ne laissent accessibles aux adultes - femelle 
féconde et ouvrières - que leur partie antérieure et surtout leur région buccale. Et les nymphes restent enfermées dans l'alvéole de la larve, qui est alors clos. Nous disposons, pour ces deux groupes d'Insectes sociaux, de données concernant les relations larves-adultes, en particulier l'alimentation des larves. Les échanges alimentaires entre larves et ouvrières, dans la société de Guêpes, ont donné lieu à une série de travaux, (Montagner, 1963 ; Ishay ef al., 1968, 1969, 1972 ; cf. Spradbery, 1973) montrant, par exemple, l'importance capitale des régurgitations larvaires pour la survie même des adultes, donc de la société. Mais il ne semble pas que ces relations (limitées, nous venons de le voir, du fait même des alvéoles) aient donné lieu à des analyses éthologiques développées et s'attachant au détail des postures et des actes qui accompagnent les échanges.

Chez les Fourmis au contraire, les larves ne sont jamais logées dans des alvéoles individuels ; elles sont ainsi accessibles par toute leur surface aux soins des ouvrières. Les larves les plus jeunes (premier stade) restent souvent accolées aux groupes d'œufs. Dès le second stade, elles sont le plus souvent réparties sur le sol ou sur les parois des chambres constituant le nid, accrochées au substrat par des poils fourchus, parfois par des pseudopodes glutineux. Les relations entre larves et adultes sont beaucoup plus variées, plus fréquentes et plus complexes que chez les Abeilles et les Guêpes sociales. Elles ont aussi donné lieu à beaucoup plus d'études analytiques. Quant aux nymphes, dans un grand nombre d'espèces elles sonf encloses dans un cocon, mais celui-ci estl'objet de soins indispensables (exposé de Jaisson, dans le présent Colloque); celles de beaucoup d'autres espèces restent nues; elles sont léchées et transportées sans cesse par les adultes, femelle fondatrice ou bien ouvrières.

Pour ces deux raisons - complexité plus grande des relations couvain-adultes, développement des recherches analytiques -, c'est sur les relations entre adultes et stades jeunes dans les sociétés de Fourmis que l'accent sera mis dans les pages qui suivent.

II faut reconnaître d'emblée que l'étude des facteurs physiologiques susceptibles d'intervenir de manière déterminante dans ces relations entre jeunes et adultes est encore peu développée pour les sociétés de Fourmis ; beaucoup moins que, par exemple, l'étude de la relation parents-jeunes chez les Mammifères, pour lesquels l'acquis est déjà très important et les recherches se développent (Poindron, 1980). Nous reviendrons sur ce point en fin d'exposé.

Par contre, nous disposons d'une masse considérable de données sur la forme de ces comportements inter-individuels, et sur leurs variations d'une espèce à l'autre, inégalement évoluées (Le Masne, 1953 ; Sudd, 1967 ; Wilson, 1971 ; sans compter de nombreux travaux monographiques mais utilisant l'analyse comparée). Une étude comparative se montre fructueuse, en particulier par les indications assez claires qu'elle apporte sur l'évolution des relations entre jeunes et adultes dans des espèces très diverses mais qui constituent certainement un ensemble homogène, monophylétique.

Un long chapitre serait nécessaire pour évoquer ces données comparatives ef leurs conséquences évolutives (Le Masne, 1953). J'indiquerai seulement que - comme 
nous l'avons noté plus hauł pour les Termites $\left({ }^{2}\right)$ - lorsqu'on passe, chez les Fourmis, des formes les plus primitives aux espèces les plus évoluées, les relations entre adultes ef stades jeunes sont de plus en plus étroites, et l'on voit dans le détail de ces relations se développer toujours plus la dépendance du couvain par rapport aux adultes et la complexité des relations qui unissent individus immatures et adultes.

Certes, aucune larve de Fourmi n'est indépendante, en particulier pour son alimentation : apode, elle attend forcément des adultes l'apport de nourriture dans le nid où elle est confinée. Cependant, les larves des espèces les plus primitives (Myrmecia ou Amblyopone) sont capables de déplacements restreints dans les loges du nid souterrain, et d'un accès parfois autonome aux proies déposées là par la femelle ou les ouvrières (Haskins, 1950 ; Gotwald et Lévieux, 1972 ; Le Masne, observation personnelle sur les Promyrmecia d'Australie). Les larves de Manica sont aptes, par une flexion du thorax hors du plan sagittal, à saisir parfois une proie déposée à côté d'elles (Le Masne et Bonavita, 1969) : reste d'un caractère archaïque, dans ce genre qui, par plusieurs de ses caractères comportementaux, se montre relativement primitif au sein d'une sous-famille évoluée ? Pour la quasi-łotalité des autres espèces de Fourmis, au contraire, les déplacements autonomes des larves sont nuls ou négligeables, en tout cas jamais orientés. Ce sont les adultes qui doivent déposer la nourriture solide (fragments de proie ou de graines) directement sur les larves, et sur leur bouche même. Ou bien, comme je l'ai noté pour Aphaenogaster subterranea, les ouvrières déposent les larves sur la nourriture, les disposant en rang sur une proie volumineuse.

Quelques faits d'ordre général donnent déjà son originalité au « modèle Fourmis » des relations entre jeunes et adultes.

La succession, avant l'état adulte, de siades très différents (œufs, larves, nymphes, l'ensemble constituant le couvain) augmente d'emblée la complexité de ces relations - même si l'on pense au cas des Oiseaux, chez qui les soins donnés aux œufs constituent, bien sûr, une étape capitale, ou bien à l'évolution individuelle très importante qui conduit, chez les Mammifères, du nouveau-né à l'individu sub-adulte.

D'autre part il existe à l'évidence, entre la larve et l'adulte de Fourmi, des différences énormes dans la structure anatomique, l'équipement sensoriel, les actions et les réactions comportementales; ces différences sont bien sûr bequcoup plus grandes que celles qui existent, par exemple, entre la brebis ef son agneau... Ce fait global entraîne d'importantes conséquences en ce qui concerne le système de communication qui unit, surtout lors des échanges de nourriture, des êtres de même espèce mais si différents : les activités, gestuelles et autres, qui précèdent ou accompagnent ces échanges ne pourront pas être semblables à celles que l'on observe lors des échanges alimentaires entre adultes (très développés chez les Insectes sociaux, contrairement aux Vertébrés supérieurs). Il s'agit là, certes, d'une donnée connue de chacun, mais qui doit être présente à l'esprit dans toute éfude de ce genre.

( $\left.{ }^{2}\right)$ Et ce rapprochement n'est pas fortuit. Il s'agit du phénomène couramment appelé convergence évolutive, et qu'on nommerait peut-être mieux évolution parallèle (cf. Le Masne, 1952, 1973, 1978). 
Une troisième différence générale (signalée dans l'exposé de Jaisson) entre Vertébrés supérieurs et Insectes sociaux, en ce qui concerne les relations entre jeunes et adultes : les adultes chargés des soins des jeunes appartiennent ici à plusieurs catégories très distinctes ; les soins du couvain sont assurés - de manière successive en général, mais parfois simultanément - non seulement par la ou les femelles fécondes, lors de la fondation de la société, mais aussi par les ouvrières dans les sociétés plus âgées. Et les membres de ces deux «castes » femelles (de même que, chez les Termites, le couple royal et les ouvriers) montrent des différences considérables dans la taille, la structure anatomique et les aptitudes physiologiques.

Cependant - sauf quelques différences à cet égard entre femelles et ouvrières (par exemple l'absence, le plus souvent, d'apport de proies lors de la fondation) les soins donnés au couvain sont dans les deux cas très semblables. Il est remarquable, pour prendre un exemple extrême, de voir les larves des Atta (Fourmis champignonnistes) soignées successivement ef de la même manière par l'énorme femelle fondatrice, longue de plusieurs centimètres, puis par les premières ouvrières, qui ne mesurent que quelques millimètres $\left(^{3}\right)$.

Une fois qu'elle a été relayée par ses ouvrières dans ses tâches de «nourrice », la femelle fondatrice cesse en général définitivement toute activité de soin du couvain. Mais on conna ît quelques cas (Wilson, 1971) où elle se montre capable de reprendre cette tâche, quand la société est privée expérimentalement de ses ouvrières.

On voit toutes les possibilités d'analyse offertes par une telle organisation sociale et quelle est alors la complexité des relations entre jeunes et adultes.

Sans ignorer la complexité des relations parentales chez les Vertébrés supérieurs, il semble que les soins donnés au couvain présentent chez les Fourmis une multiplicité et une diversité encore plus grandes, et qui ne sont pas seulement liées au nombre des stades immatures successifs, évoqué plus haut. Enumérons seulement une partie de ces activités de soins (Le Masne, 1953) :

- maniement des cufs (dans les mandibules de la femelle, puis des ouvrières), au moment de la ponte, puis transport des œufs et répartition dans le nid, soit isolément (Fourmis très archaīques du groupe des Myrmeciinae), soit plus souvent en amas compacts ;

- léchage des œufs;

- maniement des larves, qui sont souvent « classées 》 selon la taille, et séparées des œufs et des nymphes ou des cocons; au cours de ces opérations, les larves, pourvues de poils bifurqués ou de pseudopodes dorsaux, sont disposées sur les parois du nid de manière précise, la face ventrale restant accessible aux soins ;

- transport des larves, des œufs et des nymphes en différents points du nid, en fonction des variations journalières de la fempérature des chambres, ou bien lors d'un trouble brusque apporté au nid, ou encore dans les « déménagements » collectifs liés à des changements de l'emplacement du nid ;

$\left.{ }^{3}\right)$ Il est possible de négliger ici le cas des soldats de Termites, caste bien individualisée, ou des « soldats » de Fourmis, qui sont seulement des ouvrières de grande taille, avec une tête volumineuse : les uns et les autres participent peu, ou pas du tout, aux soins du couvain. 
- alimentation des larves à l'aide de fragments (de proies, de graines, etc.) déposés sur chacune; un découpage des proies en morceaux de taille variée précède ce nourrissage des larves ; cette préparation de l'aliment solide destiné aux larves peut comporter aussi un léchage des fragments, avec éventuel rejet de salive par l'ouvrière ; - régurgitation d'aliments solides, provenant du jabot des ouvrières, dans la bouche des larves (Le Masne, 1953 ; Provost, communication personnelle), à l'exclusion, en général, des larves du premier stade ;

- dégorgement, dans la bouche des larves, d'aliments liquides en provenance du jabot des ouvrières ou de leurs glandes salivaires; c'est là l'aliment principal des très jeunes larves (avec la consommation d'œufs, souvent importante) ; mais il est octroyé aussi, en général, aux larves plus âgées ;

- léchage des larves, au cours de séquences souvent très longues ; ces léchages sont indispensables à la survie des larves : lorsqu'on veut élever des larves de Fourmis indépendamment des ouvrières, on constate que ce nettoyage, à l'aide d'un pinceau fin ou de papier filtre, est tout à fait nécessaire ;

- enlèvement des excreta larvaires, qui sont ensuite déposés sur le sol, ou consommés, qu'il s'agisse d'excreta liquides en provenance des tubes de Malpighi, ou bien de l'excrément solide unique qui est rejeté à la fin de la vie larvaire (lorsque, avant la nymphose, l'intestin moyen, jusqu'alors clos à l'arrière, entre en communication avec l'intestin postérieur) ;

- assistance donnée aux larves lors des mues ;

- assistance lors de la confection du cocon : la larve file les premiers fils de soie sur des matériaux divers (sable, grains de terre...) que les ouvrières ont entassés sur elle ; une fois le cocon filé (ou même déjà après tissage de la couche la plus externe) les ouvrières le débarrassent entièrement de ces matériaux ; en l'absence de cette activité des ouvrières, et pour toutes les espèces que j'ai étudiées, la nymphe se forme cependant, mais reste nue ; dans les espèces peu évoluées - très carnivores - elle est alors consommée par les ouvrières (Haskins et Haskins, 1951 ; Le Masne, 1953) ; dans certains genres évolués (Lasius, Formica) les nymphes nues (qui peuvent apparaître aussi dans la nature, pour des raisons non connues) sont souvent respectées par les ouvrières, et donnent des adultes normaux ;

- assistance lors de l'éclosion de l'adulte hors de l'exuvie nymphale : souvent présente, cette aide ne paraît pas toujours indispensable ;

- assistance pour l'ouverture du cocon : constante ef apparemment indispensable chez les Fourmis évoluées (Camponotus, Lasius, etc.), elle semble moins nécessaire chez les espèces plus archaïques (Ponérines).

Et cette énumération n'est pas complète...

Sans doute faut-il souligner aussi l'importance de la réciprocité des échanges de substances entre larves et adultes, chez les Fourmis, Outre le liquide malpighien et l'excrément intestinal unique, mentionnés plus haut, les larves de Fourmis fournissent aux adultes, par la bouche, des liquides en provenance des glandes salivaires, ou peut-être parfois du tube digestif. De même, le léchage des larves par les adultes entraîne l'absorption de sécrétions cutanées. 
L'importance quantitative de ces transmissions de substances, allant des larves aux adultes, semble cependant moins grande que celle des aliments fournis par les adultes aux larves.

Rien, jusqu'ici, n'indique l'existence, dans les rapports entre adultes et couvain chez les Insectes sociaux, de relations préférentielles rappelant celles qui sont si développées chez certains Vertébrés supérieurs (Poindron, 1980).

Certes (puisqu'il n'existe plus guère de biologiste ou d'éthologiste qui pose en principe une opposition complète entre Vertébrés et Invertébrés évolués) il faut rester attentif à toute possibilité de ce genre. D'ailleurs des groupes préférentiels existeraient, en certains cas, dans les échanges alimentaires entre adultes, chez les Fourmis ; et des faits de reconnaissance individuelle ont été notés chez des Crustacés supérieurs (Pagure, Ecrevisse : Hazlett et al., 1965 ; Bovbjerg et al., 1971).

Cependant de telles relations préférentielles sont très peu probables, vis-à-vis des larves; et cela non seulement à l'intérieur d'une société donnée, mais aussi entre sociétés différentes (appartenant à la même espèce) : les larves sont facilement transférées d'une société à l'autre, expérimentalement, ou bien « razziées » par les ouvrières d'une société dans le nid d'une autre $\left({ }^{4}\right)$.

Dans les pages qui précèdent sont énumérées les tâches multiples qui constituent les «soins du couvain» par les adultes de Fourmis, et la fourniture de substances par les larves. Mais rien, ou presque, n'a encore été indiqué sur les interactions qui annoncent et accompagnent ces activités. En fait, nous disposons d'informations précises sur les acłes qui précèdent et préparent ou accompagnent l'alimentation des larves par les ouvrières, mais aussi le rejeł d'urine par les larves.

Avant chaque «repas» larvaire, les deux partenaires - larve et adulte effectuent des gesies et des actes bien définis, variables selon l'état physiologique des individus, et qui représentent à coup sûr un échange de stimulations et de réponses (Le Masne, 1953), au même titre que dans la relation parents-jeunes chez des Oiseaux ou des Mammifères.

La position et les mouvements d'appendices de l'ouvrière, lors du nourrissage d'une larve, ont une forme bien déterminée. La «réponse » de la larve aux contacts antennaires de l'ouvrière sur sa région antérieure comporte un redressement de la tête, ou de la tête et du thorax, qui porte sa bouche vers celle de l'ouvrière. Cette réponse peut ne pas apparaître (larve non affamée, sans doute) et l'ouvrière va alors « offrir » la nourriture à d'autres larves $\left({ }^{5}\right)$.

( $\left.{ }^{4}\right)$ Tandis que l'étude des relations entre adultes appartenant à des sociétés différentes permet de mettre en évidence (Le Masne, 1952, 1973 ; Provost, 1979) tantôł la possibilité d'adoption d'individus étrangers, ou de fusion de sociétés distinctes, tantôt une fermeture de la société, parfois très stricte.

$\left.{ }^{5}\right)$ Les ouvrières ne semblent pas capables de distinguer à quel stade d'un probable cycle alimentaire se trouve une larve donnée ; seule la réponse positive (ou son absence) les renseigne, Brian (1956) a confirmé mes observations sur ce nourrissage par la « méthode des essais et erreurs ». 
Les larves, de leur côté, peuvent « solliciter » la nourriture en soulevant la tête, lors du passage d'une ouvrière nourrice sur un « champ » de larves fixées au substrat. On peut parler de «sollicitation » : elle entraîne en effet une réponse spécifique, l'arrêt de l'ouvrière et l'octroi de nourriture. Cette activité de la larve se retrouve chez toutes les espèces étudiées, primitives ou évoluées. Elle n'esł peut-être qu'une agitation générale de la larve affamée et mise en éveil, mais elle constitue pour l'ouvrière nourrice un stimulus touł à fait significatif.

L'élevage expérimental de larves indépendamment des ouvrières - qui est peu aisé... - permet, dans une certaine mesure, l'analyse des stimulations qui précèdent les échanges, et qu'effectuent normalement les ouvrières (Le Masne, loc. cit.).

Tout comportement se modifie au cours de la vie de l'individu, et se réalise par un phénomène de développement - au même titre que toute structure ou toute fonction. Ce développement du comportement (éthogenèse, selon le terme heureux forgé par Jaisson) doit pouvoir être mis en évidence, si la vie de l'individu n'est pas trop courte. S'agissant des Fourmis, on sait depuis longtemps que la division du travail entre ouvrières est souvent fonction de leur âge (polyéthisme selon l'âge). Et l'inactivité relative des ouvrières nouvellement écloses, leur faible pigmentation même, leur accès progressif aux tâches des adultes mûrs, indiquaient l'existence d'un dévelcppement du comportement de ces Insectes, au cours des premiers jours de la vie imaginale. Heyde (1924) avait déjà montré que les réponses de la jeune ouvrière de Fourmis aux stimulations de ses compagnes passent par une série de stades intermédiaires. Schneirla $(1933,1941,1952)$ voyait dans l'acquisition des activités imaginales un véritable apprentissage. J'ai signalé également (1951) l'importance de ces faits, que j'observe aussi chez une Fourmi qui appartient à une sous-famille peu évoluée.

Les travaux de Jaisson (1973, et son exposé dans le présent Colloque) ont clairement analysé ce développement du comportement en ce qui concerne les soins donnés aux cocons par les ouvrières de Formica ; ils ont démontré qu'il s'agit d'un processus - présentant nombre de caractéristiques du phénomène d'empreinte - de familiarisation aux cocons avec lesquels l'ouvrière est en contact dans les deux premières semaines de sa vie adulte ; les stimulus olfactifs spécifiques fournis par la nymphe à travers la soie du cocon jouent là le rôle le plus important. Le Moli et Passetti (1977) ont confirmé, sur une autre espèce de Formica, les résultats de Jaisson et apporté quelques compléments.

On le voit, nous disposons, pour les sociétés de Fourmis, de beaucoup de données concernant la forme des relations entre adultes et individus jeunes (œufs, larves, nymphes ou cocons), ef l'évolution de ces relations, des espèces primitives aux plus évoluées. Nous connaissons un certain nombre de faits relatifs aux stimulations significatives qui précèdent ou accompagnent ces interactions, en particulier les échanges alimentaires entre larves et adultes. L'étude du développement de ces conduites interindividuelles (éthogenèse) est, nous venons de le voir, en progrès actif. 
Par contre, les mécanismes physiologiques associés à ces conduites inter-individuelles sont à peu près inconnus, chez les Fourmis - surtout si nous comparons à l'étendue des connaissances acquises sur le déterminisme endocrinien des relations parentales chez les Vertébrés supérieurs. Cette situation devrait pouvoir s'améliorer, étant donné le large développement des résultats dont nous disposons en ce qui concerne les régulations endocriniennes chez beaucoup d'autres Insectes ( ${ }^{6}$ ).

Je voudrais souligner, à cet égard, à quel point la biologie de la femelle féconde de Fourmi, et celle des ouvrières, offrent à l'étude un modèle remarquable par le nombre de phases successives qu'elle comportent - phases comportementales qui dépendent presque sûrement d'uétats endocriniens» successifs ou cycliquement répétés. Une énumération rapide de ces phases est à cet égard démonstrative :

- au début de sa vie adulte, la femelle vraie est tout à fait immature, y compris pour la pigmentation, et cela durant plusieurs jours, voire plusieurs semaines ;

- elle mène ensuite une vie active, se déplaçant et s'alimentant (ou étant nourrie par les ouvrières), mais sans sortir encore du nid ni montrer la moindre activité sexuelle ;

- l'activité sexuelle n'apparaît - el pour quelques heures - que lors du vol nuptial ;

- sortie du nid à cette occasion, la femelle, dans beaucoup d'espèces, va cesser toute activité sociale, et vivre solitaire durant des mois, claustrée dans une logette close, qu'elle a creusée sous terre en général ; elle ne s'alimente pas et « consomme » ses muscles alaires devenus inutiles ;

- survient la ponte, groupée en une courte période, parfois en deux ou trois «couvées » au cours de la phase solitaire ;

- la femelle - nous l'avons indiqué plus haut - exerce alors toutes les activités que reprendront plus tard les ouvrières : construction du nid, soin du couvain (avec approvisionnement seulement chez des espèces primitives : cf. plus haut) ;

- dès l'éclosion des premières ouvrières, elle va, de manière progressive mais en général rapide, cesser toutes ces activités, qu'elle laisse à ses filles; elle se limite, outre son nourrissage par les ouvrières, à la ponte et à des activités de toilette (auxquelles les ouvrières participeront largement d'ailleurs, surtout si la différence de taille est grande entre elles et la femelle). Elle conserve cependant quelques relations avec le couvain chez les espèces primitives, et aussi chez celles des formes évoluées où la femelle féconde n'est pas beaucoup plus grande que les ouvrières (Le Masne, 1953) ; - la ponte, pendant plusieurs années successives (10 à 20 ans pour certaines espèces), va survenir par « couvées » successives, séparées par des intervalles plus ou moins longs. Ce rythme de ponte peut, par l'intermédiaire de l'activité des larves, commander de manière cyclique toute la vie de la société : le cas le plus spectaculaire est, à cet égard, celui des Fourmis nomades Eciton où ce processus commande l'alternance de phases nomades et sédentaires qui caractérise leur vie sociale (Schneirla, 1933, 1952...); avec des variations comportementales de moindre envergure, le même phénomène doit pouvoir être mis en évidence chez d'autres Fourmis ;

(6) Et l'étude des facteurs endocriniens qui sont associés aux phases successives du comportement parental est entamée, pour les Forficules par exemple (Pierre, $1979 a, b$ ). 
- à ce cycle de ponte relativement court (quelques semaines) se superpose un cycle annuel, avec en général - en région tempérée du moins - une inactivité hivernale et une reprise de la ponte cyclique au printemps ;

- cette énumération, abrégée, des phases d'activité comportementale de la femelle féconde devrait être complété par une liste, un peu moins longue, concernant l'ouvrière.

Il apparaît évident - du moins cette direction de recherche doit-elle être suggérée - que des changements de l'activité des différentes parties du système endocrinien des Fourmis, en particulier des femelles fécondes, peuvent être associés à cette succession de phases comportementales. L'étude des relations entre le système endocrine rétro-cérébral de la femelle féconde de Fourmis et sa physiologie ovarienne est entreprise (Délye, 1973 ; Palma, 1975 ; Palma et Délye, 1979) ; elle montre, par exemple, une corrélation entre l'évolution des cellules neuro-sécrétrices et le fonctionnement ovarien. Mais l'étude expérimentale reste à faire. Surtout, pour notre propos, l'analyse des relations entre système endocrine, ovaire et phases du comportement parental de ces femelles fécondes reste à entreprendre.

Colloque « La relation parentale » Nouzilly, France, juin 1979.

\section{Références}

ALIBERT J., 1959. Les échanges trophallactiques chez le Termite à cou jaune (Calotermes flavicollis Fabr.) étudiés à l'aide du phosphore radioactif. C. R. Acad. Sci. Paris, 248, 1040-1042.

ALIBERT J., 1960. Les échanges trophallactiques entre Termites sexués ef larves de jeunes fondations de colonies et de sociétés plus âgées (Calotermes flavicollis Fabr.). C. R. Acad. Sci. Paris, 250, 4205-4207.

ALIBERT J., 1963. Echanges trophallactiques chez un Termite supérieur. Contamination par le phosphore radio-actif de la population d'un nid de Cubitermes fungifaber. Insectes soc. 10, 1-2.

ALIBERT J., 1969. Influence de la société et de l'individu sur la trophallaxie chez Calotermes flavicollis Fabr. et Cubitermes fungifaber. (Colloque sur l'Effet de groupe chez les animaux, Paris, 1967). Coll. int. C.N.R.S., 173, 237-288.

BOVBJERG R. V., STEPHEN S. L., 1971. Behavioral changes in crayfish with increased population density. Bull. ecol. Soc. Amer., 52, 37-38.

BRIAN M. V., 1956. Group form and causes of working inefficiency in the Ant Myrmica rubra L. Physiol. Zcol., 29, 173-194.

BUCHLI H., 1950. Recherches sur la fondation et le développement des nouvelles colonies chez le Termite lucifuge. Phys. comp. OEcol., 2, 145-160.

CHAUVIN R., 1968. Traité de biologie de l'abeille. Masson, Paris, 2 (Système nerveux, comportement et régulations sociales).

DÉLYE G., 1973. Neurosécrétion et reproduction chez les reines de Camponotus vogus Scop. Proc. 7e Congr. I.U.S.S.I., Londres, 88-91.

GÖSSWALD K., KLOFT W., 1958. Radioaktive Isotope zur Erforschung an staatenlebenden Insekten. Umschau, 58, 743-745.

GOTWALD W. H. Jr., LÉVIEUX J., 1972. Taxonomy and biology of a new west african Ant belonging to the genus Amblyopone (Hymenoplera : Formicidae). Ann. entom. Soc. Amer., 65, 383-396.

GRASSÉ P.-P., 1949. Ordre des Isoptères ou Termites. Traité de Zoologie. Masson, Paris, 9, $408-544$.

GRASSÉ P.-P., NOIROT C., 1945 . La transmission des Flagellés symbiotiques et les aliments des Termites. Bull. biol. Fr. Belg., 79, 273-292. 
GRASSÉ P.-P., NOIROT C., 1951. La sociotomie : migration et fragmentation de la termitière chez Anoplotermes ef Trinervitermes. Behaviour, 3, 146-166.

HASKINS C. P., HASKINS E. F., 1950. Note on the biology and social behavior of the archaic Ponerine Ants of the genera Myrmecia and Promyrmecia. Ann. Entom. Soc. Amer., 43, 461-491.

HASKINS C. P., HASKINS E. F., 1957. Note on the method of colony foundation of the Ponerine Ant Amblyopone australis Erichson. Amer. Midland Not., 45, 432-455.

HAZLETT B. A., BOSSERT W. H., 1965. A statistical analysis of the agressive communications systems of some hermit crabs. Anim. Behav., 13, 357-373.

HEYDE K., 1924. Die Entwicklung der psychischen Fähigkeiten bei Ameisen und ihr Verhalten bei abgeänderten biologischen Bedingungen. Biol. Zentralblatt, 44, 623-654.

ISHAY J., IKAN R., 1968. Food exchange between adults and larvae in Vespo orientalis F. Anim. Behav., 16, 298-303.

ISHAY J., IKAN R., 1969. Gluconeogenesis in the oriental hornet Vespa orienfalis F. Ecology, 49, 169171.

ISHAY J., LANDAU E. M., 1972. Vespa larvae send out rhytmic hunger signals. Nature, London, 237, 286-287.

JAISSON P., 1973. L'imprégnation dans l'ontogenèse du comportement de soins aux cocons chez la jeune Fourmi rousse (Formica polyctena Först.). Behaviour, 52, 1-37.

JAISSON P., 1980. Les relations parentales chez les Hyménoptères sociaux. Reprod. Nutr. Dévelop., 20, $771-772$.

KRAFFT B., HOREL A., 1980. Comportement maternel ef relations mères-jeunes chez les Araignées. Reprod. Nutr. Dévelop., 20, 757-758.

LE MASNE G., 1951. Echanges de nourriture, trophallaxie ef transports mutuels chez les Fourmis. In P. GRASSÉ, Traité de Zoologie, X, fasc. 2, 1104-1119, Masson, Paris.

LE MASNE G., 1952. Classification et caractéristiques des principaux types de groupements sociaux réalisés chez les Invertébrés. (Coll. int. Structure et Physiologie des Sociétés animales, Paris, 1950). Coll. int. C.N.R.S., 34, 19-70.

LE MASNE G., 1953. Observations sur les relations entre le couvain ef les adultes chez les Fourmis. Bull. sect. fr. U.I.E.I.S., 1, et Ann. Sci. nat., Zool., 11'e sér., 15, 1-56.

LE MASNE G., 1973. Sociétés animales. Encyclopaedia Universalis, Paris, 15, 53-64.

LE MASNE G., 1978. Les méthodes d'étude de l'évolution des comportements. (Coll. Méthodologie comp. des Sciences, Dijon 1975). Rev. Quest. Scient., Namur, 149, 357-396.

LE MASNE G., BONAVITA A., 1969. La fondation des sociétés selon un type archaïque par une fourmi appartenant à une sous-famille évoluée. C. R. Acad. Sci. Paris, 269, 2373-2376.

LE MOLI F., PASSETTI M., 1977. The effect of early learning on recognition, acceptance and care of cocoons in the Ant Formica rufa L. Atti Soc. ital. Sc. nat., Milan, 118, 49-64.

MCMAHAN E. A., 1963. A study of Termite feeding relationships, using radio-isotopes. Ann. entom. Soc. Amer., 56, 74-82.

MCMAHAN E. A., 1969. Feeding relationships and radioisotope techniques. Biology of Termites, KRISHNA K., WEESNER F. M., Acad. Press, N. Y., 1, 387-406.

MONTAGNER H., 1963. Etude préliminaire des relations entre les adultes ef le couvain chez les Guêpes sociales du genre Vespa, au moyen d'un radio-isotope. Insectes soc., 10, 153-165.

NOIROT C., 1952. Les soins et l'alimentation des jeunes chez les Termites. Bull. sect. fr. U.I.E.I.S., 1, et Ann. Sci. nat., Zool., 11e sér., 14, 405-414.

PALMA G., 1975. Recherches sur le déterminisme neuro-endocrinien de la reproduction chez les reines de Camponotus. Th. 3e Cycle, Univ. Provence, Marseille, 91 pp.

PALMA-VALLI G., DÉLYE G., 1979. Contrôle neuro-endocrinien de la ponte chez les reines de Camponotus lateralis Olivier. Insectes soc., (sous presse).

PIERRE J. S., 1979a. Le cycle parental de Labidura riparia (Insectes Dermaptère) : contribution à l'étude de certains déterminants neuro-endocriniens. Th. $3^{e}$ Cycle, Univ. Rennes, 129 pp.

PIERRE J. S., 1979b. Effet de l'allatectomie sur la fin du cycle parental chez Labidura riparia Pallas (Dermaptères Labiduridae). Biol. Comport., 4, 219-226.

POINDRON P., LE NEINDRE P., RAKSANYI I. TRILLAT G., ORGEUR P., 1980. Importance of the characteristics of the young in the manifestation and establishment of maternal behaviour in sheep. Reprod. Nuir. Dévelop., 20, 817-826. 
PROVOST E., 1979. Etude de la fermeture de la société de Fourmis chez diverses espèces de Leptothorax ef chez Camponotus lateralis. C. R. Acad. Sci. Paris, 288, 429-432.

SCHNEIRLA T. C., 1933. Some important features of Ant learning. Z. vergl. Physiol., 19, 439-452. SCHNEIRLA T. C., 1941. Social organisation in Insects, as related to individual function. Psychol. Rev., 48, 465-486.

SCHNEIRLA T. C., 1952. Basic correlations and coordinations in Insect societies, with special reference to Ants. (Coll. int. Structure et Physiologie des Sociétés animales, Paris, 1950). Coll. int. C.N.R.S., 34, 247-269.

SPRADBERY J. R. Wasps, an account of the biology and natural history of solitary and social wasps. Sidgwick and Jackson, Londres, 408 pp.

SUDD J. H., 1967. An introduction to the behaviour of Ants. Arnold, Londres, 200 pp.

VANCASSEL M., FORASTÉ M., 1980. Le comportement parental des Dermaptères. Reprod. Nutr. Dévelop., 20, 759-770.

WILSON E. O., 1971. The Insect Societies. Belknap Press, Harvard Univ. Press, Cambridge, Mass., $548 \mathrm{pp}$. 